\title{
Relação de pareceristas ad hoc (2015-2016)
}

Educação e Pesquisa agradece aos especialistas abaixo relacionados, que colaboraram com a revista no período de out. 2015 a out.2016.

Adão José Peixoto
Ademir Macedo Nascimento
Adilson Odair Citelli
Adir Casaro Nascimento
Adla Betsaida Martins Teixeira
Adna de Almeida Lopes
Adolfo Samuel de Oliveira
Adriana Bauer
Adriana Bortoletto
Adriana Marcondes Machado
Adriano Correia Silva
Adriano Henrique Nuernberg
Adriano Vargas Freitas
Afonso Henriques
Agustín Adúriz-Bravo
Aida Maria Monteiro Silva
Alda Junqueira Marin
Alessandra Carbonero Lima
Alessandra Seabra
Alex Branco Fraga
Alexandre Simão Freitas
Alfredo José da Veiga-neto
Alice Fushako Itani
Allan Moreira Xavier
Alvaro Chrispino
Álvaro Luiz Moreira Hypolito
Amarilio Ferreira Junior
Amaury Cesar Moraes
Amélia Cristina Abreu Artes
Ana Angelita Costa Neves da Rocha
Ana Celuta Fulgêncio Taveira
Ana Cláudia Balieiro Lodi
Ana Claudia Urban
Ana Dorziat
Ana Laura Godinho Lima Paula dos Santos Malheiros
Ana Paula Leivar Brancaleoni
Ana Luiza Bustamante Smolka Jesus da Costa
Ana Márcia Silva
Ana Maria Duarte Gomes
Ana Maria Eyng
Ana Maria Fonseca Almeida
Ana Maria Galvão
Ana Maria Petraitis Liblik
Ana Padawera Saul
Anillela Cavaliere
Ana Carneiro Renesto
Aliveira Corti
Ana Panas
Anas

Ana Paula Machado Velho

Ana Paula Martinez Duboc

Ana Paula Soares da Silva

Ana Sueli Teixeira de Pinho

Ana Valéria de Figueiredo da Costa

Ana Waleska Pollo Campos Mendonça

Anderson Ferrari

André Bocchetti

André de Macedo Duarte,

Andréa Barbosa Gouveia

Andrea Coelho Lastoria

Andrea Cristina Filatro

Andréa Cristina Pavão Bayma

Ângela Magda Rodrigues Virgolim

Angela Medeiros Santi

Ângela Pereira Teixeira Victoria Palma

Angélica Monteiro

Ângelo Ricardo de Souza

Anna Augusta Sampaio de Oliveira

Antonia Terra Calazans

Antonio Barros de Brito Junior

Antônio Carlos Brolezzi

Antonio Carlos Gaeta

Antonio Carlos Rodrigues Amorim

Antonio Cipriano Gonçalves

Antonio Hilario Aguilera Urquiza

Antonio Joaquim Severino

Antonio Munarim

Antonio Simplicio de Almeida Neto

Antonio Takao Kanamaru

Artemis Augusta Mota Torres

Áurea Maria Guimarães

Beatriz de Basto Teixeira

Beatriz Maria Boéssio Atrib Zanchet,

Belmira Amélia Oliveira Barros Bueno

Benerval Pinheiro Santos

Bianca Cristina Correa

Bruno Pucci

Carla Alexandra Moita Minervino

Carla Biancha Angelucci

Carla Fabiana Cazella

Carla Hernandez Silva

Carla Mari Rodrigues

Carlos Antônio Giovinazzo Jr.

Carlos Augusto Candêo Fontanini

Carlos Bauer

Carlos Frederico Bernardo Loureiro

Carlos Roberto Cury

Carlos Roberto Jamil Cury

Carmen Fernandez

Carmen Lúcia Soares

Carmen Mazepa Ballão
Carol Reid

Carolina Carolina Picchetti Nascimento

Carolina Faria Alvarenga

Carolina Tamayo Osorio

Cássia Geciauskas Sofiato

Caterine Vila Fagundes

Cecilia Guarnieri Batista

Cecilia Hanna Mate

Celso João Ferretti

Celso Roberto Perez

Charles dos Santos Guidotti

Cintya Regina Ribeiro

Cipriano Carlos Luckesi

Clarice Seixas Duarte

Claudia Araújo da Cunha

Claudia Machado

Cláudia Maria Mendes Gontijo

Cláudia Pereira Vianna

Cláudia Rosa Riolfi

Cláudia Valentina Assumpção Galian

Claudio Almir Dalbosco

Cláudio Antônio Tordino

Cláudio José de Almeida Mello

Cláudio Marques Martins Nogueira

Claus Dieter Stobäus

Cleoni Maria Barbosa Fernandes

Cristiane Coppe de Oliveira

Cristiane Machado

Cristiane Maria Cornelia Gottschalk

Cristiane Maria Galdino de Almeida

Cristiano Perius

Cristina Cinto Araújo Pedroso

Cynthia Paes de Carvalho

Daisa Teixeira

Daniel Mill

Daniela Aparecida Eufrásio

Dario Fiorentini

David Michael Bloome

Debora Cristina Jeffrey

Débora de Barros Silveira

Débora Martins Artiaga

Delcele Mascarenhas Queiroz

Deller James Ferreira

Denise Balarine Cavalheiro Leite

Denise Meyrelles de Jesus

Denise Rosana da Silva Moras

Denise Rosana Moraes

Denize de Aguiar Xavier Sepulveda

Dennis de Oliveira

Diana Souza Carvalho

Diana Vidal

Dislane Zerbinatti Moraes 
Douglas Eliesler Justen

Ducéria Tartuci

Dulcinéia Ester Pagani Gianotto

Edda Curi

Edna Antonia Mattos Morais Andrade

Edna Oliveira

Edson Caetano

Edson Schroeder

Eduardo José Manzini

Elania Oliveira

Elba Siqueira de Sá Barreto

Elenice de Souza Lodron Zuin

Elenice Maria Cammarosano Onofre

Elenilce Gomes Oliveira

Eliane Marta Teixeira Lopes

Elie George Guimarães Ghanem Júnior

Elione Maria Nogueira Diógenes

Elisabeth Mattos

Elizabeth Santos Braga

Elizeu Clementino de Souza

Émerson de Pietri

Eneida Oto Shiroma

Erenice Natalia Soares de Carvalho

Erivaldo Vieira da Silva

Ermelinda Moutinho Pataca

Ester Maria Dreher Heuser

Evandro Alves

Fabiana Augusta Alves Jardim

Fabiana de Oliveira

Fabiana Fabiana de Oliveira

Fabiana Jardim

Fabiana Silva Fernandes

Fabiana Soares Fernandes

Fabiany de Cassia Tavares Silva

Fabio de Barros Silva

Fábio Hoffmann Pereira

Fábio Mallart Moreira

Fabíola Stolf Brzozowski

Faleiro Wender Faleiro

Felipe da Silva Triani

Fernando Henrique Protetti

Fernando Seffner

Filipe Ceppas de Carvalho e Faria

Flávia Inês Schilling

Flavine Assis de Miranda

Flavinês Rebolo

Flavio Henrique Albert Brayner

Francisca Izabel Pereira Maciel

Frederico Carlos Frederico Bernardo Loureiro

Gabriela Miranda Moriconi

Gabriela Rodella de Oliveira

Geide Rosa Coelho

Geraldo Leão

Geraldo Magela Pereira Leão

Gilda Cardoso Araújo

Giselle Cristina Martins Real

Giselle Martins dos Santos Ferreira

Giselle Watanabe

Glades Tereza Felix
Gladis Teresinha Taschetto Perlin

Gladys Beatriz Barreyro

Glauco Nunes Souto Ramos

Glícia Salviano Gripp

Graça Regina Franco da Silva Reis

Graziela Serroni Perosa

Guaracira Gouvêa

Guilherme do Val Toledo Prado

Gustavo Ferreira Costa Lima

Helder Boska de Moraes Sarmento

Helena Altmann

Helena Coharik Chamlian

Helena Singer

Heloisa Helena Pimenta Rocha

Hustana Maria Vargas

Inês Signorini

Ione Ribeiro Valle

Ione Vasques-Menezes

Isabel Alice Lelis

Isabel C.M. Carvalho

Isabel de Oliveira e Silva

Isabel Gretel María Eres Fernández

Isabel Melero Bello

Isabel Oliveira Silva

Itamar Mendes Silva

Jacques Rocha Velloso

Jacques Velloso

Jaqueline Batista de Oliveira Costa

Jaqueline Kalmus

Jefferson Mainardes

Jeronimo Jorge Cavalcante Silva

Jill Koyama

João Airton de Matos Pontes

João Batista Siqueira Harres

João Marcos Mateus Kogawa

Jocélio Teles dos Santos

Joice de Espindola

Jorge Dorfman Knijnik

José Alves da Silva

José Augusto Brito Pacheco

José Benedito Almeida Júnior

José Carlos Libâneo

José Carlos Oliveira Costa

José Damiro de Moraes

José Geraldo Silveira Bueno

José Luiz Magalhães de Freitas

José Marcelino de Rezende Pinto

José Márcio Augusto Oliveira

Jose Marcio Barros

José Márcio Pinto de Moura Barros

José Maurício Paiva Andion Arruti

José Pedro Boufleuer

José Sérgio Fonseca de Carvalho

Jose Simoes de Almeida Junior

José Vaidergorn

Juarez Tarcisio Dayrell,

Juliana Aparecida Matias Zechi

Juliana Crespo Lopes

Julio Groppa Aquino
Jussara Biagini

Karina Maldonado Pagnez

Karina Soledad Maldonado Molina Pagnez

Karla de Almeida Chediak

Kátia Maria Abud

Katia Morosov Alonso

Kátia Silva Cunha

Kátia Stocco Smole

Kazumi Munakata

Lazara Cristina da Silva

Leda Scheibe

Leila Pio Mororó

Lenilda Cordeiro de Macêdo

Léo Barbosa Nepomuceno

Leonardo Maciel Moreira

Letícia Jovelina Storto

Lidiane de Souza Assante

Lighia Brigitta Horodynski-Matsushigue

Ligia Gomes Elliot

Lígia Paula Couto

Lílian do Valle

Liliane Ferrari Giordani

Lisandra Ogg Gomes

Lisani Geni wachholz Coan

Lívia Andrade Coelho

Lourdes Helena da Silva

Lourdes Marcelino Machado

Lourdes Maria Werle de Almeida

Lourenilson Leal de Sousa

Luci Banks Leite

Lúcia Emília Bruno

Lúcia Gouvêa Pimentel

Lucia Isaltina Clemente Leão

Lucia Pereira Leite

Luciana Conrado Martins

Luciana Massi

Luciana Pacheco Marques

Luciano Mendes Faria Filho

Lucídio Bianchetti

Luciene Regina paulino Tognetta

Luís Gustavo Alexandre da Silva

Luis Marcelo Carvalho

Luís Miguel Carvalho

Luísa Margarida Cagica Carvalho

Luiz Alberto Boing

Luiz Alberto Rezende

Luiz Antônio Cunha

Luiz Bezerra Neto

Luiz Marcelo de Carvalho

Lygia de Sousa Viégas

Magda Becker Soares

Manoel Lima Cruz Teixeira

Manoel Oriosvaldo Moura

Marcelo A Ribeiro

Marcelo Afonso Ribeiro

Marcelo El Khouri Buzato

Márcia Buss-Simão

Márcia Denise Pletsch

Márcia Ondina Vieira Ferreira 
Marcia Regina Oliveira Andrade

Márcio Costa

Márcio Danelon

Marco Antonio Coelho Bortoleto

Marco Antonio Leandro Barzano

Marcos Antonio de Almeida

Marcos Antonio dos Santos Reigota

Marcos Antônio Lorieri

Marcos Cezar de Freitas

Marcos Francisco Martins

Marcos Freisleben Zorzal

Marcos Garcia Neira

Marcos José da Silveira Mazzotta

Marcos Nascimento Magalhães

Marcos Sidnei Pagotto-Euzebio

Marcos Tarcísio Masetto

Marcus Vinicius da Cunha

Marcus Vinicius Fonseca

Margarida Dias Pocinho

Margarida Pocinho

Mari Valicheski Ferrari,

Maria Alice Nogueira

Maria Amélia Rosário Santoro Franco

Maria Ângela Borges Salvadori

Maria Aparecida Morgado

Maria Aparecida Viggiani Bicudo

Maria Cândida Müller

Maria Carmo Lacerda Peixoto

Maria Cecilia Sanches

Maria Ciavatta

Maria Clara Di Pierro

Maria Cláudia Dal'Igna

Maria Cláudia Santos Lopes de Oliveira

Maria Cristina Cavaleiro

Maria Cristina Machado Kupfer

Maria Cristina Martins

Maria Cristina Martins Penido

Maria Cristina Menezes

Maria Cristina Pansera de Araújo

Maria Cristina Soares de Gouvea

Maria da Graça Jacintho Setton

Maria das Mercês Ferreira Sampaio

Maria de Assunção Lima de Paulo

Maria de Fátima Barbosa Abdalla

Maria de Fatima Felix Rosar

Maria de Lourdes Macena de Souza

Maria de Lourdes Spazziani

Maria do Carmo de Lacerda Peixoto

Maria do Carmo de Sousa

Maria do Rosário Zara Figueiredo Tripodi

Maria do Socorro Leal Lopes

Maria do Socorro Xavier Batista

Maria Elizabeth Bianconcini Trindade Morato

Pinto Almeida

Maria Emília Caixeta de Castro Lima

Maria Eulina Pessoa de Carvalho

Maria Fátima Barbosa Abdalla

Maria Fatima Felix Rosar

Maria Fátima Salum Moreira
Maria Glória Marcondes Gohn

Maria Graça Jacintho Setton

Maria Helena Bertolini Bezerra

Maria Helena Camara Bastos

Maria Helena Maria Helena Oliveira Gonçalves

Augusto

Maria Helena Silveira Bonilla

Maria Inês Ribas Rodrigues

Maria Isabel Almeida

Maria Isabel da Cunha

Maria Isabel de Almeida

Maria Isabel Petry Kehrwald

Maria Isabel Ramalho Ortigão

Maria José Fontelas Rosado Nunes

Maria José Maria Albuquerque da Silva

Maria José Pereira Monteiro Almeida,

Maria Júlia Canazza Dall'Acqua

Maria Letícia Barros Pedroso Nascimento

Maria Livia Tommasi

Maria Lúcia Rodrigues Muller

Maria Luisa Furlan Costa

Maria Luiza Belloni

Maria Margarida Pereira de Lima Gomes

Maria Núbia Barbosa Bonfim

Maria Regina Kawamura

Maria Renata da Cruz Duran

Maria Rosa Lombardi

Maria Rosário Longo Mortatti

Maria Silvia Cárnio

Maria Stella Bresciani

Maria Teresa Eglér Mantoan

Maria Teresa Gonzaga Alves

Maria Teresa Vianna Van Acker

Maria Vieira Silva

Mariana Paladino

Marieta Gouvêa de Oliveira Penna

Marieta Lúcia Machado Nicolau

Marilda Gonçalves Dias Facci

Marília Gomes de Carvalho

Marília Pontes Sposito

Marina Jorge da Silva

Marines Boncoski Brizola

Mário Henrique Gomes

Marisa Bittar

Marisa Grigoletto

Marisa Ribeiro Teixeira Duarte

Marizabel Kowalski

Marize Cisneiro da Costa Reis

Marizete Lucini

Marlene Ribeiro

Marli Elisa Dalmaso Afonso D'André

Marli Lúcia Tonatto Zibetti

Marlúcia Menezes de Paiva

Marta Kohl de Oliveira

Marta Maria do Amaral Azevedo

Marta Maximo Pereira

Martha Marandino

Martha Tristão

Mauro Betti
Mauro Guimarães

Maxim Repetto

Máximo Daniel Lamela Adó

Mercedes Marcilese

Michele Tomoko Sato

Michelle Jaber da Silva

Michelle Prazeres Cunha

Miriam Abramovay

Miriam Cardoso Utsumi

Miriam Struchiner

Mitsuko Aparecida Makino Antunes

Moema de Poli Teixeira

Moema de Rezende Vergara

Monalisa Muniz

Mônica Caldas

Mônica Castagna Molina

Mônica Maria Guimarães Savedra

Mônica Pereira do Sacramento

Moysés Kuhlmann Júnior

Murilo José de Resende

Nadir Zago

Nalú Farenzena

Neide Luzia de Rezende

Nesdete Mesquita Corrêa

Neuza Bertoni Pinto

Newton Bryan

Newton Duarte

Nilton Mullet Pereira

Noeli Prestes Padilha Rivas

Núbio Mafra

Nuria Hanglei Cacete

Ocimar Munhoz Alavarse

Oldimar Pontes Cardoso

Olgaíses Cabral Maués

Olgamir Amância Ferreira de Paiva

Oscar João Abdounur

Otavio Aloísio Maldaner

Pablo Francisco Di Leo

Pablo Silva Machado Bispo dos Santos

Paolo Nosella

Patrícia Coelho Costa

Patrícia Rodrigues Chaves da Cunha

Paula Arcoverde Cavalcanti

Paula Baptista Jorge Louzano

Paula Louzano

Paula Ramos de Oliveira

Paulino de Jesus Francisco Cardoso

Paulo Eduardo Ramos

Paulo Fraga da Silva

Paulo Henrique Fernandes Silveira

Paulo Roberto Menezes Lima JR.

Paulo Romualdo Hernandes

Paulo Takeo Sano

Pedro Angelo Pagni

Pedro Carlos Pereira

Pedro Demo

Percival Tavares da Silva

Rafael de Brito Dias

Ramofly Bicalho dos Santos 
Raquel Goulart Barreto

Raquel Nery Lima Bezerra

Regina Célia Couto

Reginaldo Fernando Carneiro

Reginaldo Fernando Carneiro

Reinaldo Matias Fleuri

Renata Cristina Oliveira Barrichelo Cunha

Renata Simões Duarte

Renato Cancian

Renato José de Oliveira

Ricardo Desidério da Silva

Ricardo Ribeiro Terra

Rinaldo Voltolini

Rita Cássia Pereira Lima

Rita de Cássia Bortoletto-Santos

Rita Márcia Magalhães Furtado

Roberto Muniz Barretto de Carvalho

Roberto Paulo Machado Lopes

Rodrigo Pelegrini Ratier

Rodrigo Saballa de Carvalho

Rodrigo Travitzki

Rogério de Almeida

Romélia Mara Alves Souto

Romilda Teodora Ens

Romualdo Portela Oliveira

Ronaldo Marcos de Lima Araujo

Roni Cleber Dias de Menezes

Ronice Muller Quadros

Rosa Fátima Souza

Rosa Maria Bueno Fischer

Rosa Maria Ferreira da Silva

Rosa Maria Hessel Silveira

Rosalba Maria Cardoso Garcia

Rosane Aragón

Rosangela Aparecida Hilário

Rosangela Fritsch

Roselene Fatima Coito

Rosemeire de Araújo Rangni

Rute Estanislava Tolocka

Ruth Mercado Maldonado

Saddo Ag Almouloud

Salete Linhares Queiroz

Salomão Barros Ximenes
Samir Haddad

Samuel Mendonça

Sandra Carli

Sandra Eli Sartoreto de Oliveira Martins

Sandra Eliza Vielmo

Sandra Maria Sawaya

Sandra Maria Zákia Lian Sousa

Sandra Mónica Figueiredo Figueiredo Oliveira

Sandra Regina Oliveira Garcia

Sandra Unbehaum

Sergio Antonio Carlos

Sergio Neves Dantas

Sheila Daniela Medeiros dos Santos

Shirlei Rezende Sales

Sibele Cazelli

Sibila Luft

Sidney Jard da Silva

Silas Borges Monteiro

Silvana Aparecida de Souza

Silvana Pezzi

Silvia Cristina Yannoulas

Silvia Dias Alcântara Machado

Silvia Kimo Costa

Silvia Lúcia Bigonjal Braggio

Silvia Maria Cintra da Silva

Silvio Gallo

Simone Albuquerque da Rocha

Simone Costa Nunes

Simone Eliza do Carmo Lessa

Simoni Tormöhlen Gehlen

Sirlene Aparecida Aarão

Sônia Cristina Soares Dias Vermelho

Sônia Cristina Vermelho

Sonia Maria Duarte Greego

Sônia Maria Portella Kruppa

Sônia Maria Vieira Negrão

Sônia Regina dos Santos Teixeira

Sônia Teresinha Sousa Penin

Soraia Napoleão Freitas

Souzana Mizan

Stela Conceição Bertholo Piconez

Sueli Salles Fidalgo

Susana Beatriz Sacavino
Susana Graciela Pérez Barrera Pérez Suzana Feldens Schwertner

Suzana lanna Burnier Coelho

Tânia Mara Cruz

Tania Maria Esperon Porto

Tânia Maria Rechia Schroeder

Tatiane Cosentino Rodrigues

Terciane Ângela Luchese

Teresa Helena Schoen

Thais Mere Marques Aveiro

Thomaz Edson Veloso da Silva

Tizuko Morchida Kishimoto

Ubiratan D'Ambrosio

Ulysses Rocha Filho

Umberto de Andrade Pinto

Valdete Côco

Valéria Amorim Arantes

Valéria Peixoto de Alencar

Valter Carabetta Júnior

Vanda Moreira Machado Lima

Vanessa Ferraz Almeida Neves

Vânia de Vasconcelos Gico

Vânia Maria de Araújo Passos

Vanina Costa Dias

Vera Maria Ferrão Candau

Vera Maria Nigro de Souza Placco

Vera Teresa Valdemarin

Veronica Gesser

Victor Leonardi

Vinício Macedo Santos

Viviane Briccia do Nascimento

Viviane Castro Camozzato

Wagner Bandeira Andriol

Walter Omar Kohan

Wanda Maria Junqueira de Aguiar

Wania Clemente Castro

Wania Lacerda

Wiara Rosa Alcântara

Wildson Luiz Pereira dos Santos

Will Ribamar Mendes Almeida

Wivian Weller

Yara Araújo Ferreira Guimarães

Zilma Moraes Ramos Oliveira 